\title{
Clinical Outcomes of Critically Ill Patients Using Inhaled Nitric Oxide (iNO) during Intrahospital Transport
}

\author{
Leonid Koyfman, ${ }^{1}$ Omri Simchon, ${ }^{1}$ Anna Koyfman, ${ }^{2}$ Shoshana Mushaev, ${ }^{3}$ \\ Benjamin F. Gruenbaum, ${ }^{4}$ Ron Gal, ${ }^{1}$ Michael Friger, ${ }^{5}$ Natan Arotsker, ${ }^{1}$ Alexander Zlotnik, \\ Moti Klein, ${ }^{1}$ and Evgeni Brotfain ${ }^{1}{ }^{1}$ \\ ${ }^{1}$ Department of Anesthesiology and Critical Care, General Intensive Care Unit, Soroka Medical Center, \\ Ben-Gurion University of the Negev, Beer Sheva, Israel \\ ${ }^{2}$ Department of Radiology, Meir Medical Center, Kfar Saba, Israel \\ ${ }^{3}$ Department of Internal Medicine, Soroka Medical Center, Ben-Gurion University of the Negev, Beer Sheva, Israel \\ ${ }^{4}$ Department of Anesthesiology and Perioperative Medicine, Mayo Clinic, Jacksonville, FL, USA \\ ${ }^{5}$ Department of Public Health, Faculty of Health Sciences, Ben-Gurion University of the Negev, Beer Sheva, Israel
}

Correspondence should be addressed to Evgeni Brotfain; bem1975@gmail.com

Received 29 December 2020; Revised 16 March 2021; Accepted 27 April 2021; Published 6 May 2021

Academic Editor: Fred A. Luchette

Copyright (c) 2021 Leonid Koyfman et al. This is an open access article distributed under the Creative Commons Attribution License, which permits unrestricted use, distribution, and reproduction in any medium, provided the original work is properly cited.

\begin{abstract}
Critically ill patients with severe hypoxemia are often treated in the intensive care unit (ICU) with inhaled nitric oxide (iNO). These patients are at higher risk when they require intrahospital transportation. In this study, we collected clinical and laboratory data from 221 patients who were hospitalized in the general ICU and treated with iNO at Soroka Medical Center, Israel, between January 2010 and December 2019. We retrospectively compared the 65 patients who received iNO during intrahospital transportation to the 156 patients who received iNO without transportation. Among critically ill patients who were transported while being administered iNO, only one patient had an adverse event (atrial fibrillation) on transport. We found that maximal iNO dosage during ICU stay, duration of mechanical ventilation, and percent of vasopressor support were the only independent risk factors for ICU mortality in both study groups. No difference in primary outcome of ICU mortality rate was found between the critically ill patients treated with iNO during intrahospital transportation and those who were treated with iNO but not transported during the ICU stay. We anticipate that this study will advise clinical decision-making in the ICU, especially when treating patients who are administered iNO.
\end{abstract}

\section{Introduction}

It is widely known that patients in the intensive care unit (ICU) are at increased risk for complications when transported within the hospital for diagnostic or therapeutic procedures [1-3]. Parmentier-Decrucq et al. [3] recorded and analyzed 262 intrahospital transportations of 184 patients who needed a computed tomography (CT) scan. A total of $45.8 \%$ of the patients suffered from complications and $16.8 \%$ of the patients suffered major complications, including oxygen desaturation, extubation, hemodynamic instability, and increased vasopressor dose. Out of all the complications, $16.8 \%$ were patientrelated and $32.8 \%$ were equipment-related.
Critically ill patients with severe hypoxemia, often caused by acute respiratory distress syndrome (ARDS), are at particular risk from complications of hospital transportation. These patients require advanced mechanical ventilation with a high mean airway pressure and positive end-expiratory pressure (PEEP), a high percentage of oxygen, deep sedation, and muscle relaxation $[4,5]$. Some of these severely affected patients are commonly treated with inhaled nitric oxide (iNO).

A large percentage of ICU patients treated with iNO develop rebound hypoxemia and pulmonary hypertension following immediate withdrawal of iNO. Thus, when discontinuing iNO, it is important to slowly wean patients off 
the gas [1]. Any transportation of this patient subpopulation outside of the ICU is complex and potentially life-threatening as interruption of iNO delivery, for even a short period of time, can be dangerous [1].

Despite the common use of iNO in intensive care units throughout the world, few reports have examined the use of continuous iNO therapy in critically ill patients during intrahospital transportation while in the ICU [6-8]. In previously published data, there have been reports of continuous iNO for transfer of adult critical care patients with severe hypoxemia to a tertiary center [4]. Flatten et al. presented a case report of a critically ill patient with severe ARDS who successfully underwent intrahospital transport and major surgery while being treated with iNO [9]. Also, several studies describe successful usage of iNO during interhospital transport of preterm and full-term newborns suffering from persistent pulmonary hypertension [10-12]. We retrospectively assessed and compared the clinical outcomes of critically ill patients who were treated with iNO during their ICU stay and during intrahospital transportation to the outcomes of patients who received iNO only during their ICU stay.

\section{Methods}

The study was conducted at Soroka Medical Center, a 1,000bed tertiary care university hospital located in Israel. We retrospectively collected the clinical and laboratory data of all the critically ill patients who were treated with iNO while hospitalized in the general ICU at Soroka between January 2010 and December 2019. Clinical information was retrieved from our computerized Registration Information System (MetaVision ${ }^{\circledR}$, iMDsoft ${ }^{\circledR}$ Israel and "OFEK" Electronic Data). The study was approved by the Human Research and Ethics Committee at Soroka University Medical Center (RN -0237-19-SOR).

2.1. Inclusion Criteria. All critically ill patients aged $\geq 18$ years who were hospitalized for more than 72 hours in the ICU at Soroka Medical Center between January 2010 and December 2019 and who were treated with iNO during their ICU stay were eligible for inclusion in the study.

2.2. Exclusion Criteria. The following patients were excluded from the study: critically ill patients who were hospitalized in preparation for organ donation and patients whose medical records contained insufficient data.

2.3. Variables and Measures. The demographic data included the patients' admission diagnoses, the presence or absence of comorbidities, previous chronic drug treatment, and the duration of the patients' ICU and hospital admissions. The following laboratory findings were documented at the time of admission to the ICU: hemoglobin level and white blood cell count, as well as blood glucose, urea, and creatinine levels. The following parameters were also recorded: length of mechanical ventilation (MV); ventilation parameters such as $\mathrm{PaO}_{2} / \mathrm{FiO}_{2}$ ratio, $\mathrm{PEEP}, \mathrm{FiO}_{2}$, and lung compliance; the eventual success or failure of weaning from $\mathrm{MV}$ and days without MV (ventilator-free days); use of vasopressors and steroids during ICU hospitalization; parameters related to the treatment of iNO such as length of iNO treatment and iNO dose; parameters related to iNO intrahospital transport including the frequency of transport during the ICU hospitalization period, iNO dose during transport, place of transport, duration of transport, and the presence or absence of complications during transport and their type; echocardiographic features such as left and right ventricle function or evidence of pulmonary hypertension; Acute Physiology and Chronic Health Evaluation-II (APACHE-II) and Therapeutic Intervention Scoring System (TISS) scores; and in-ICU and in-hospital mortality rates.

2.4. Definitions. The severity of the patients' illnesses and the presence or absence of multiorgan failure were evaluated within 24 hours of ICU admission in accordance with the patients' APACHE-II and TISS scores.

2.5. Study Groups. All study participants were treated with iNO. After retrospective analysis of their clinical and laboratory data, the study patients were divided into two groups: patients who were not transported outside of the ICU during their hospitalization period (Group 1) and patients that were transported outside of the ICU during iNO treatment during their hospitalization period (Group 2). Transportation was defined as any intrahospital transport of a patient outside of the ICU for any diagnostic or therapeutic procedure purposes. Intrahospital transportation included cardiorespiratory monitoring, portable mechanical ventilation machine (using similar background respiratory parameters), and mobile iNO device and tank.

2.6. Inhaled Nitric Oxide (iNO) Therapy. Inhaled nitric oxide (iNO) therapy was administered to mechanically ventilated, sedated, and paralyzed critically ill patients with refractory hypoxemia due to severe acute respiratory distress syndrome (ARDS) $\left(\mathrm{PO}_{2} / \mathrm{FiO}_{2}\right.$ ratio $\left.<100, \mathrm{FiO}_{2} 1.0, \mathrm{PEEP}>12 \mathrm{cmH}_{2} \mathrm{O}\right)$. The starting dose of iNO was $20 \mathrm{ppm}$.

2.7. Primary Endpoint. Duration of mechanical ventilation during the ICU stay was defined as the primary endpoint.

2.8. Secondary Endpoints. The following secondary endpoints were defined: intrahospital mortality, the duration of the patients' ICU and hospital stay, the duration of MV while in the ICU, success or failure of weaning from MV, and changes in iNO connectivity or respiratory parameters while on intrahospital transport.

2.9. Statistical Analysis. Statistical analysis was performed in three steps: The first step is descriptive statistics. Variables that are normally distributed are described by the mean and standard deviation (such as age, weight, and more). 
TABLE 1: The demographics, underlying conditions, admission diagnoses, laboratory data, and therapeutic management during the ICU stay.

\begin{tabular}{|c|c|c|c|}
\hline & Group $1^{*}(n=156)$ & Group 2* $(n=65)$ & $P$ value** \\
\hline Age, years $\left(\right.$ mean $\left.\pm S D^{b}\right)$ & $50.92 \pm 19.826$ & $50 \pm 18.822$ & 0.751 \\
\hline Weight, $\mathrm{kg}($ mean $\pm \mathrm{SD})$ & 80.763 & 90.523 & 0.655 \\
\hline Male gender, no. (\%) & $112 / 156(72.4 \%)$ & $47 / 65(72.3 \%)$ & 0.985 \\
\hline \multicolumn{4}{|l|}{ Admission diagnosis, no. (\%) $)^{* * *}$} \\
\hline Sepsis & $47 / 156(30.1 \%)$ & $28 / 65(43.1 \%)$ & 0.174 \\
\hline Multiple traumas & $67 / 156(43.6 \%)$ & $24 / 65(36.9 \%)$ & 0.179 \\
\hline Other & $41 / 156(26.3 \%)$ & $13 / 65(20 \%)$ & 0.97 \\
\hline \multicolumn{4}{|l|}{ ICU scores at admission (units, mean \pm SD) } \\
\hline $\mathrm{APACHE}^{\mathrm{c}}$ score & $26.49 \pm 4.7$ & $25.8 \pm 4.28$ & 0.34 \\
\hline TISS $^{\mathrm{d}}$ score & $29.81 \pm 9.51$ & $27.57 \pm 5.54$ & 0.07 \\
\hline \multicolumn{4}{|l|}{ Laboratory data during iNO treatment in ICU } \\
\hline $\mathrm{WBC}^{\mathrm{e}}(1000$ cells $/ \mu \mathrm{L}$, mean $\pm \mathrm{SD})$ & $16.582 \pm 9.523$ & $14.986 \pm 8.652$ & 0.245 \\
\hline Hemoglobin (g\dL) & $11.528 \pm 3.033$ & $11.420 \pm 2.586$ & 0.801 \\
\hline Serum urea $(\mathrm{mmol} / \mathrm{L})$ & $58.32 \pm 53.086$ & $54.29 \pm 43.592$ & 0.590 \\
\hline Serum creatinine $(\mathrm{mmol} / \mathrm{L})$ & $1.626 \pm 2.892$ & $1.286 \pm 0.903$ & 0.354 \\
\hline Blood glucose level (mg/dL) & $156.79 \pm 62.036$ & $161.85 \pm 61.739$ & 0.581 \\
\hline \multicolumn{4}{|l|}{ Underlying conditions, no. (\%) } \\
\hline $\mathrm{CIHD}^{\mathrm{f}^{\prime}}$ & $36 / 156(23.1 \%)$ & $13 / 65(20.1 \%)$ & 0.83 \\
\hline $\mathrm{COPD}^{\mathrm{g}}$ & $24 / 156(15.4 \%)$ & $20 / 65(30.8 \%)$ & 0.02 \\
\hline $\mathrm{HTN}^{\mathrm{h}}$ & $31 / 156(19.9 \%)$ & $18 / 65(27.7 \%)$ & 0.04 \\
\hline Other $^{\mathrm{i}}$ & $64 / 156(41.7 \%)$ & $14 / 65(21.5 \%)$ & 0.03 \\
\hline \multicolumn{4}{|c|}{ Therapeutic management and diagnostic tests during ICU stay, no. $(\%)^{* * * *}$} \\
\hline Vasopressor support & $119 / 156(76.3 \%)$ & $46 / 65(69.2 \%)$ & 0.275 \\
\hline Steroid treatment & $87 / 156(56.4 \%)$ & $40 / 65(61.5 \%)$ & 0.482 \\
\hline \multicolumn{4}{|l|}{ ECHO study findings } \\
\hline Left ventricle dysfunction (moderate/severe) & $47 / 156(30.1 \%)$ & $20 / 65(29.7 \%)$ & 0.297 \\
\hline Right ventricle dysfunction & $50 / 156(32 \%)$ & $18 / 65(28 \%)$ & 0.616 \\
\hline Pulmonary hypertension (moderate/severe) & $62 / 156(40.3 \%)$ & $21 / 65(33.3 \%)$ & 0.757 \\
\hline
\end{tabular}

${ }^{*}$ Group 1: critically ill patients who were not transported outside of the ICU during their hospitalization period. Group 2: critically ill patients who were transported outside of the ICU during iNO treatment during their hospitalization period. ${ }^{* *}$ Data are considered statistically significant if $P<0.05 .{ }^{* * *}$ Other diagnoses include intracerebral hemorrhage, cardiogenic shock, eclampsia, SAH, NSTEMI, and pulmonary edema. ${ }^{* * * *}$ Vasopressor support: norepinephrine continuous infusion; steroid treatment: concomitant hydrocortisone administration. ${ }^{a}$ ICU: intensive care unit. ${ }^{b}$ SD: standard deviation. ${ }^{c}$ APACHE: Acute Physiology and Chronic Health Evaluation on admission to ICU. ${ }^{\mathrm{d}}$ TISS: Therapeutic Intervention Scoring System on admission to ICU. ${ }^{\mathrm{e}} \mathrm{WBC}$ : white blood cells. ${ }^{\mathrm{f}} \mathrm{CIHD}$ : chronic ischemic heart disease. ${ }^{\mathrm{g}} \mathrm{COPD}$ : chronic obstructive pulmonary disease. ${ }^{\mathrm{h}} \mathrm{HTN}$ : chronic hypertension. ${ }^{\mathrm{I}}$ Other: diabetes mellitus II, dyslipidemia, alcohol abuse, fat embolism, and pulmonary embolism.

Variables that were normally distributed are described by median and range (e.g., time). Qualitative variables such as gender are described by their percentage distribution. The second step is univariate analysis. At this point, each variable was compared to all independent variables. Comparison of a dependent variable with quantitative variables that are normally distributed as weight/age was done by means of a $t$ test for independent samples. A comparison of a dependent variable with quantitative variables that are not normally distributed was done by means of a Mann-Whitney test. Comparison of a dependent variable with quality variables used the independent chi-squared test or Fisher's exact test as needed. The third step is multivariate mortality testing. For this testing, we used logistic regression. The model includes an independent variable and also variables that are significant in the second stage and/or clinically important. We also examined possible interactions between variables. For multivariate survival analysis, we used Cox regression. Data analysis was processed with SPSS Version 25. The significance level in multivariate analysis was set at 0.05 . Data were analyzed using IBM SPSS 22 (NY, USA) and Epi Info 3.5.1 (CDC, GA, USA).

\section{Results}

A total of 221 patients received inhaled nitric oxide (iNO) while hospitalized in the general ICU during the study period. 10 patients had insufficient data records and were excluded from the study. A total of 221 study patients were divided into two groups on the basis of a retrospective analysis of their transportation outside of the ICU.

The patients' demographic, clinical, and laboratory data and the details of their ICU therapeutic management are presented in Table 1. There were no significant differences between the two groups in their demographic parameters (age, weight, and gender), admission diagnoses, APACHE and TISS scores, or their laboratory data (Table 1). The proportion of patients with a past history of chronic obstructive pulmonary disease (COPD) and chronic hypertension (HTN) was significantly higher in Group 2 (transported patients) than in Group 1 (nontransported patients) $(P P=0.02,0.04$, respectively, Table 1$)$. The percentages of patients treated with vasopressor support or steroid treatment or who had abnormal echocardiography findings were not different between both study groups. All 
TABLE 2: iNO treatment data and intrahospital patient transportation (Group (2)) data during the ICU stay.

\begin{tabular}{|c|c|c|c|}
\hline & Group $1^{*}(n=156)$ & Group 2* $(n=65)$ & $P$ value** \\
\hline Maximal iNO dosage during ICU stay (ppm, mean \pm SD) & $35.08 \pm 15.618$ & $40.95 \pm 18.488$ & 0.017 \\
\hline iNO dosage during intrahospital transportation $(\mathrm{ppm}$, mean $\pm \mathrm{SD})$ & NA & $32.51 \pm 14.181$ & - \\
\hline iNO treatment length $($ days, mean \pm SD) & $4.5 \pm 4.643$ & $9.57 \pm 10.239$ & 0.0001 \\
\hline Duration of intrahospital transportation (minutes, mean \pm SD) & NA & $116.89 \pm 10.7$ & - \\
\hline \multicolumn{4}{|l|}{ Intrahospital transport location (\%) } \\
\hline $\mathrm{CT}^{\mathrm{a}}$ & NA & $52.2 \%$ & - \\
\hline Operating room (OR) & NA & $41.8 \%$ & - \\
\hline Other (angiography, other intensive care unit) & NA & $6 \%$ & - \\
\hline \multicolumn{4}{|l|}{ Frequency of intrahospital transportation (\%) } \\
\hline One & NA & $71.6 \%$ & - \\
\hline Two & NA & $14.9 \%$ & - \\
\hline Three & NA & $4.5 \%$ & - \\
\hline
\end{tabular}

* Group 1: critically ill patients who were not transported outside of the ICU during their hospitalization period. Group 2: critically ill patients who were transported outside of the ICU during iNO treatment during their hospitalization period. ${ }^{* *}$ Data are considered statistically significant if $P<0.05 .{ }^{a} \mathrm{CT}$ : computerized tomography.

TABLE 3: Clinical outcome endpoints of both study groups (mean $\pm \mathrm{SD}$ and median).

\begin{tabular}{|c|c|c|c|}
\hline & Group $1^{*}(n=156)$ & Group 2* $(n=65)$ & $P$ value** \\
\hline Duration of $\mathrm{MV}^{\mathrm{a}}$ during $\mathrm{ICU}^{\mathrm{b}}$ stay (days, mean $\pm \mathrm{SD}$ ) & $18(2-48)$ & $24(1-62)$ & 0.078 \\
\hline Days without MV (ventilation-free days) (days, mean \pm SD) & $2.81 \pm 3.591$ & $2.95 \pm 4.488$ & 0.799 \\
\hline Patients weaned from MV, no. (\%) & $62.2 \%$ & $63.1 \%$ & 0.513 \\
\hline Length of ICU stay (days, mean \pm SD) & $22(5-52)$ & $27(4-66)$ & 0.108 \\
\hline Length of hospital stay (days, mean $\pm \mathrm{SD}$ ) & $32.5(11-75)$ & $40(9-82)$ & 0.31 \\
\hline ICU mortality (\%) & $32.1 \%$ & $29.2 \%$ & 0.68 \\
\hline \multicolumn{4}{|l|}{ Intrahospital mortality (after discharge from ICU) } \\
\hline$(\%)$ & 0 & $1.9 \%$ & 0.26 \\
\hline
\end{tabular}

${ }^{*}$ Group 1: critically ill patients who were not transported outside of the ICU during their hospitalization period. Group 2: critically ill patients who were transported outside of the ICU during iNO treatment during their hospitalization period ${ }^{* *}$ Data are considered statistically significant if $P<0.05$. ${ }^{a} \mathrm{MV}$ : mechanical ventilation. ${ }^{\mathrm{b}} \mathrm{ICU}$ : intensive care unit.

patients included in the present study were sedated, continuously paralyzed with muscle relaxants, and mechanically ventilated. Basic ventilation parameters including $\mathrm{PaO}_{2} /$ $\mathrm{FiO}_{2}$ ratio, $\mathrm{PEEP}, \mathrm{FiO}_{2}$, and lung compliance were similar in both study groups.

Analysis of the patients' iNO treatment data during the ICU stay demonstrated a significant difference in maximal iNO dosage and iNO length of treatment between the two study groups, with the Group 2 transported patients consistently showing higher iNO dose and longer iNO treatment than the Group 1 nontransported patients $(P<0.017$ and $<0.0001$, respectively, Table 2 ). Critically ill patients treated by iNO (Group (2) were transported during their ICU stay at least once (71.6\% of patients) to CT imaging and OR in the majority of cases (Table 2). Overall time of intrahospital transportation was $116.89 \pm 10.7$ minutes. One of these patients (Group 2, transported patients) developed rapid atrial fibrillation (AF) during intrahospital transportation (heart rate $>100$ beats/min) which was treated successfully by continuous amiodarone.

Routine chest X-rays after comparison to X-rays before intrahospital transportation of critically ill patients with iNO demonstrated no change in $30 \%$ of patients, while $25 \%$ had worse outcomes in radiologic criteria of ARDS. 35\% of chest $\mathrm{X}$-Rays showed radiographic improvement after intrahospital transportation. No further clinical deterioration or adverse effects were demonstrated after intrahospital transport.

The ICU and intrahospital mortality rates were similar in both groups (Table 3). There were no differences between the groups in MV, duration of their ICU stay and hospital stay, proportion of patients weaned successfully from MV, and the number of ventilation-free days (Table 3 ).

Table 4 shows the results of multivariate logistic regression analysis of Group 1 and Group 2. Maximal iNO dosage during ICU stay, duration of mechanical ventilation, and percent of vasopressor support were found to be the only independent risk factors for ICU mortality in both study groups.

\section{Discussion}

Nitric oxide (NO) is a selective pulmonary vasodilator, and it has been successfully used as a rescue therapy for severe refractory hypoxemia [7]. iNO administration reduces endogenous NO production, and therefore rapid withdrawal of iNO can cause a significant rebound pulmonary hypertension and subsequent hypoxemia. This can be avoided by gradual withdrawal $[7,8]$. iNO is administered most commonly to invasively ventilated patients.

Despite the clear clinical benefit for the improvement of oxygenation and minimal potential adverse events related to 
TABLE 4: Multivariate logistic regression analysis for risk factors for ICU mortality in critically ill patients with iNO treatment.

\begin{tabular}{lccc}
\hline & $\mathrm{OR}^{\mathrm{a}}$ & $95 \% \mathrm{CI}^{\mathrm{b}}$ of OR & $P$ value \\
\hline Maximal iNO dosage during ICU & 1.042 & $1.009-1.077$ & 0.013 \\
Duration of $\mathrm{MV}^{\mathrm{d}}$ during ICU stay (days, mean \pm SD) & 0.932 & $0.895-0.97$ & 0.001 \\
Vasopressors support $($ mean $\pm \mathrm{SD}$ ) & 64.01 & $2.095-195.5$ & 0.017 \\
\hline
\end{tabular}

${ }^{\mathrm{a}} \mathrm{OR}$ : odds ratio. ${ }^{\mathrm{b}} \mathrm{CI}$ : confidence interval. ${ }^{\mathrm{c} I C U}$ : intensive care unit. ${ }^{\mathrm{d}} \mathrm{MV}$ : mechanical ventilation.

iNO use in critically ill patients with ARDS, there is insufficient evidence that it improves patients' clinical outcome [8]. Furthermore, the difficulties arising from equipment and clinical management of critically ill patients requiring diagnostic procedures, urgent surgeries, or intrahospital relocations are associated with significant complications [2].

In the present study, we compared the clinical outcomes of two groups of critically ill patients who were treated by iNO during the ICU stay. Group 1 consisted of patients who were not transported outside of the ICU during their hospitalization period. Group 2 consisted of patients who were transported outside of the ICU while being treated with iNO during their hospitalization period. The Group 2 patients (iNO transportation group) demonstrated significantly higher iNO doses and longer iNO treatment compared to Group 1 patients (nontransported patients). Overall intrahospital transportation of critically ill patients with iNO was safe (with only one case of rapid AF) and average time of transport outside the ICU with iNO was about two hours. Moreover, $15 \%$ and $4.5 \%$ of critically ill patients were transported safely with iNO two and three times, respectively, during the ICU stay. Our findings are similar to those found in the neonatal population in newborns with severe hypoxemia refractory to conventional therapy due to congenital diaphragmatic hernia ( $\mathrm{CDH})$, PPHN, meconium aspiration syndrome, sepsis, and non- $\mathrm{CDH}$ pulmonary hypoplasia [12]. Kinsella et al. [12] observed uneventful and safe interhospital iNO transport for 25 term newborns with overall iNO dosage at about $25 \mathrm{ppm}$. Teman et al. [4] demonstrated significant improvement in oxygenation of patients with severe hypoxemia using iNO during safe transport to a tertiary care center. In the present study, the patients were treated with an even higher iNO dose (>30 ppm) during intrahospital transport.

Several recent studies have shown the beneficent perioperative use of iNO for cardiac surgery, as well as lung and cardiac transplantations [6]. Two studies by Flaatten et al. [9] and Haddad et al. [13] reported the safety of iNO use during different types of surgery for adult and pediatric populations. In the data presented here, about $42 \%$ of critically ill patients were safely transported to the OR and used iNO during surgery.

Importantly, our univariate statistical analysis did not show differences in the ICU or hospital mortality rates between the two subpopulations. Moreover, patients in both study groups had similar durations of MV, vasopressor support, ICU, and hospital stay. Furthermore, multivariate analysis demonstrated that the following factors were independent predictors of increased ICU mortality among both study groups: maximal iNO dosage during ICU stay, duration of mechanical ventilation, and percent of successful weaning from mechanical ventilation. There is not sufficient evidence in recently published literature to support that iNO reduces mortality in critically ill patients with severe ARDS $[7,8]$.

This study has several limitations, including a lack of adequate information concerning the patients' medical history. In addition, our data did not contain complete records of complications that may have taken place after intrahospital transport of severe ARDS patients treated by iNO. Furthermore, the groups that were examined were not identical and had different underlying conditions. These differences can significantly impact outcome. We anticipate that future prospective studies on this topic will correct any limitations caused by this study's retrospective design.

In the present study, we demonstrated that maximal iNO dosage during ICU stay is associated with high risk of ICU mortality in critically ill patients with severe ARDS. We suggest that this finding is correlated with severe critical illness of ARDS patients hospitalized in the ICU rather than an effect of iNO treatment on patients' clinical outcome. Further studies are needed to better understand the clinical significance of iNO treatment use during intrahospital and interhospital transportation in critically ill patients.

\section{Conclusion}

No difference in primary outcome (ICU mortality rate) was found between the ICU patients treated with iNO with intrahospital transportation and those who were not transported during the ICU stay. This study provides an insight into the utility of iNO for critically ill patients, suggesting that it does not primarily change outcomes even for patients who undergo intrahospital transportation. It might be inferred that intrahospital transfer with iNO is safe for the critically ill population. We anticipate the relevance for this study in clinical decision-making within the ICU, especially for treating critically ill patients receiving iNO.

\section{Data Availability}

The data that support the findings of this study are available from the corresponding author, Evgeni Brotfain, upon reasonable request.

\section{Ethical Approval}

The Human Research and Ethics Committee at Soroka Medical Center in Beer-Sheva, Israel, approved this study (RN-0237-19-SOR). 


\section{Disclosure}

This study was conducted as part of the requirements for MD degree from the Goldman Medical School at the Faculty of Health Sciences, Ben-Gurion University of the Negev.

\section{Conflicts of Interest}

The authors declare that no competing financial or other conflicts of interest exist.

\section{Authors' Contributions}

Dr. Leonid Koyfman participated in literature search, study design, data collection, data analysis, and data interpretation (leonidko@gmail.com). Dr. Omri Simchon participated in study design, data collection, data analysis, data interpretation, and writing (omri63014@gmail.com). Dr. Anna Koyfman participated in study design, data collection, data analysis, data interpretation, and writing (zelenenkayaa@ mail.ru). Dr. Shoshana Mushaev participated in study design, data collection, data analysis, data interpretation, and writing (evgenibr@clalit.org.il). Dr. Benjamin F. Gruenbaum participated in study design, data collection, data analysis, data interpretation, and writing (bengruenbaum@gmail.com). Dr. Ron Gal participated in the design of the study, performed the statistical analysis, and helped to revise the manuscript (rongalgat@walla.co.il). Professor Michael Friger participated in data collection and data interpretation and performed the statistical analysis (Friger@bgu.ac.il). Dr. Nathan Arotsker participated in the design of the study, performed the statistical analysis, and helped to revise the manuscript (natansky11@gmail.com). Professor Alexander Zlotnik participated in study design, data collection, data analysis, data interpretation, and writing (AleksZl@clalit.org.il). Professor Moti Klein conceived of the study, participated in its design and coordination, and helped to draft the manuscript (motik@ clalit.org.il). Professor Evgeni Brotfain participated in the design of the study and coordination and helped to draft the manuscript (bem1975@gmail.com). All authors read and approved the manuscript. Drs. Koyfman and Simchon contributed equally to the paper.

\section{References}

[1] J. Á. Monsalve-Naharro, E. Domingo-Chiva, S. García Castillo, P. Cuesta-Montero, and J. M. Jiménez-Vizuete, "Inhaled nitric oxide in adult patients with acute respiratory distress syndrome," Farm Hosp, vol. 41, no. 2, pp. 292-312, 2017.

[2] U. Beckmann, D. M. Gillies, S. M. Berenholtz, A. W. Wu, and P. Pronovost, "Incidents relating to the intra-hospital transfer of critically ill patients," Intensive Care Medicine, vol. 30, no. 8, pp. 1579-1585, 2004.

[3] E. Parmentier-Decrucq, J. Poissy, R. Favory et al., "Adverse events during intrahospital transport of critically ill patients: incidence and risk factors," Annals of Intensive Care, vol. 3, no. 1, p. 10, 2013.

[4] N. R. Teman, J. Thomas, B. S. Bryner et al., "Inhaled nitric oxide to improve oxygenation for safe critical care transport of adults with severe hypoxemia," American Journal of Critical Care, vol. 24, no. 2, pp. 110-117, 2015.

[5] F. Alessandri, F. Pugliese, and V. M. Ranieri, "The role of rescue therapies in the treatment of severe ARDS," Respiratory Care, vol. 63, no. 1, pp. 92-101, 2018.

[6] E. A. Bacha and C. A. Head, "Use of inhaled nitric oxide for lung transplantation and cardiac surgery," Respiratory Care Clinics of North America, vol. 3, no. 4, pp. 521-536, 1997.

[7] B. C. Creagh-Brown, M. J. Griffiths, and T. W. Evans, "Benchto-bedside review: inhaled nitric oxide therapy in adults," Critical Care, vol. 13, no. 3, p. 221, 2009.

[8] F. Gebistorf, O. Karam, J. Wetterslev, and A. Afshari, "Inhaled nitric oxide for acute respiratory distress syndrome (ARDS) in children and adults," The Cochrane Database of Systematic Reviews, vol. 27, no. 6, Article ID CD002787, 2016.

[9] H. Flaatten, S. Gjerde, S. Gullestad, and S. Aardal, "Surgery during inhalation with nitric oxide in a patient with severe adult respiratory distress syndrome (ARDS)," Acta Anaesthesiologica Scandinavica, vol. 40, no. 3, pp. 376-378, 1996.

[10] J. Gien, C. Nuxoll, and J. P. Kinsella, "Inhaled nitric oxide in emergency medical transport of the newborn," NeoReviews, vol. 21, no. 3, pp. e157-e164, 2020

[11] S. Tang and O. Miller, "Inhaled nitric oxide during emergency neonatal transportation," Journal of Paediatrics and Child Health, vol. 32, no. 6, pp. 539-541, 1996.

[12] J. P. Kinsella, J. Griebel, J. M. Schmidt, and S. H. Abman, "Use of inhaled nitric oxide during interhospital transport of newborns with hypoxemic respiratory failure," Pediatrics, vol. 109, no. 1, pp. 158-161, 2002.

[13] E. Haddad, S. M. Lowson, R. A. Johns, and G. F. Rich, "Use of inhaled nitric oxide perioperatively and in intensive care patients," Anesthesiology, vol. 92, no. 6, pp. 1821-1825, 2000. 